\title{
Modulating host metabolism
}

The extracellular pathogenic bacterium group A Streptococcus (GAS) requires access to host cell nutrients for successful infection and proliferation. Hanski and colleagues now show that GAS modulates host cell metabolism to induce the production and extracellular release of asparagine, which is sensed by the pathogen and promotes bacterial growth.

While investigating the in vivo conditions that activate the GAS quorum-sensing system sil (streptococcal invasion locus) the authors found that induction of this locus was facilitated by physical contact between GAS and mammalian cells but that it could also occur by indirect contact, albeit less efficiently. As the GAS toxins streptolysin S (SLS) and SLO are secreted but are more efficiently delivered into host cells during adherence, these findings suggest that SLS and SLO might be involved in sil activation. To test this hypothesis, the authors generated GAS mutants that lacked either SLS, SLO or both and found that, whereas sil was activated in the isogenic

GAS modulates

host cell

metabolism

to induce the

production and

extracellular

release of asparagine mutants, the double mutant lost this ability, which suggests that each toxin can induce sil independently of the other.

A previous study reported that SLO modulates host cellular responses, including endoplasmic reticulum (ER) stress. Hanski and colleagues went on to show that chemical inducers of ER stress could activate sil, which supports the idea that sil activity is augmented by GAS-induced ER stress in host cells. As ER stress has been linked to increased asparagine synthase (asns) expression, the authors analysed asns expression levels in GAS-infected cells. Using realtime reverse transcription PCR of infected mouse embryonic fibroblasts, an increase in the level of asns transcripts was detected compared with uninfected cells or with cells that were infected with the double mutant lacking both SLS and SLO. These data suggested that SLS- and SLO-mediated ER stress triggers increased expression of asns. Moreover, addition of extracellular asparagine to GAS cultures led to sil activation and, importantly, promoted the growth of GAS. The authors went on to show that the released asparagine is sensed by GAS via the TrxSR two-component system.

Transcriptome analysis showed that host-derived asparagine alters the expression of 311 GAS genes, whereas asparagine deficiency resulted in decreased transcription of genes that are involved in GAS proliferation. This trait was found to be preserved among different GAS strains. The authors further found that only about one-third of these asparagine-responsive genes were regulated by TrxSR, which suggests that an additional yet-to-be identified system is involved in asparagine sensing. Interestingly, the authors found that the genes encoding SLO
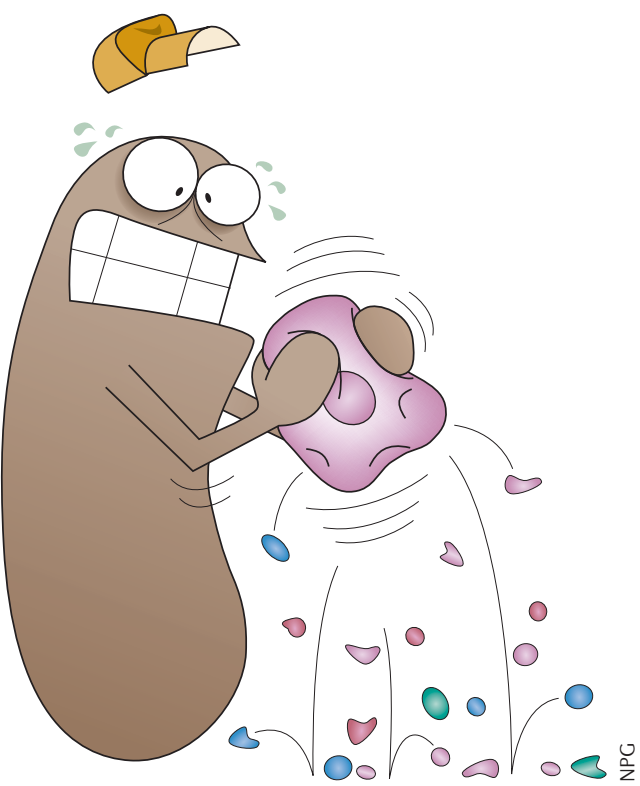

and SLS were strongly upregulated in the absence of asparagine, which suggests a mechanism whereby GAS increases streptolysin expression under asparagine-depleted conditions to cause ER stress and promote asparagine release from host cells.

This study identifies a hostpathogen interaction whereby GAS exploits host asparagine metabolism for its own benefit. Interestingly, treatment with asparaginase arrested GAS growth, which holds promise for the development of novel therapeutic strategies to control GAS infections.

Andrea Du Toit

ORIGINAL RESEARCH PAPER Baruch, M. et al. An extracellular bacterial pathogen modulates host metabolism to regulate its own sensing and proliferation. Cell 156, 97-108 (2014) 